\title{
Overweight and obesity are common in rich and poor
}

Figure 1. Overweight or obesity by sex, persons aged 16 years and over, NSW, least disadvantaged quintile, 1997 to 2011.

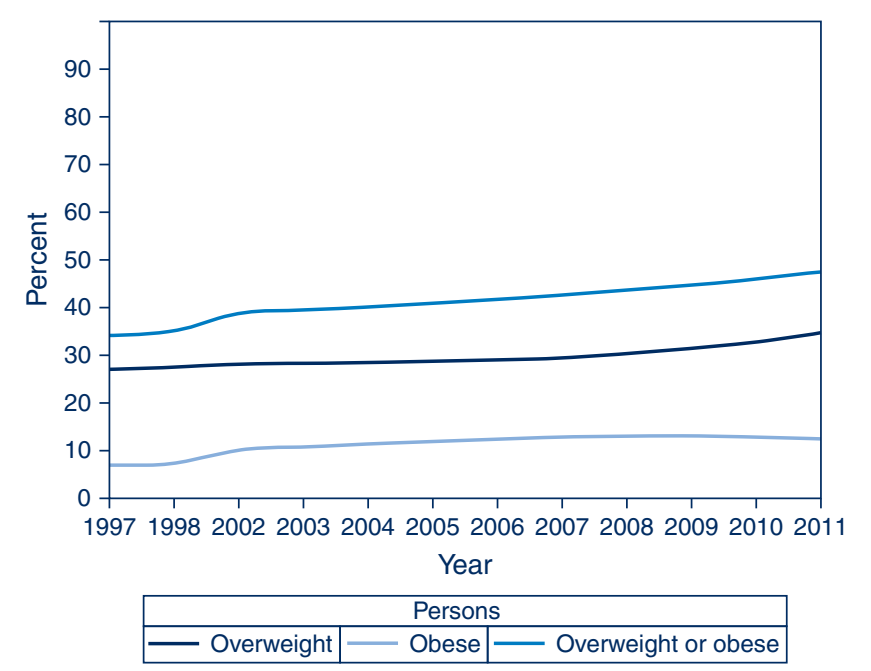

Figure 2. Overweight or obesity by sex, persons aged 16 years and over, NSW, most disadvantaged quintile, 1997 to 2011.

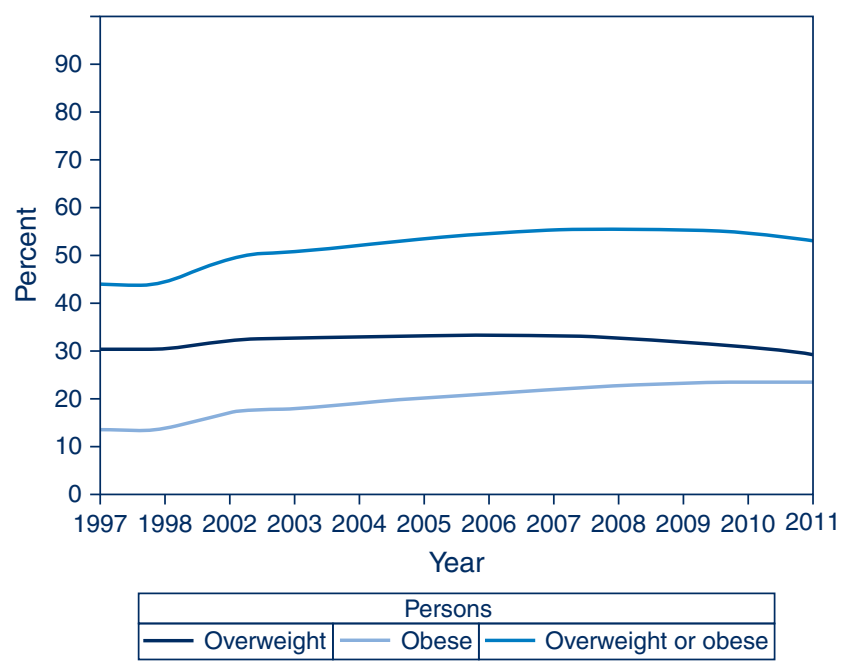

Source: NSW Adult Population Health Survey (SAPHaRI). Centre for Epidemiology and Evidence, NSW Ministry of Health.

A key performance indicator for the NSW health system is the prevalence of 'overweight or obesity' in the population. The amalgamation of the two categories of 'obese' and 'overweight' in this indicator masks differing trends in these individual categories over time. For the entire NSW population, the total category of 'overweight or obese' increased by $11.1 \%$ between 1997 and 2011. The increase for the 'obese' category alone was however $8.5 \%$ over this period and 'overweight' alone was $2.6 \%$. The above figures compare these trends in the highest socioeconomic status (SES) (least disadvantaged) quintile (Figure 1) and the lowest SES (most disadvantaged) quintile (Figure 2). These figures show that, while the rate of increase of total 'overweight and obesity' between 1997 and 2011 is similar in the two groups, the rate of increase for the 'obese' category alone was higher in the low SES group compared to the high SES group over this period. The prevalence of 'obesity' alone increased from 7.2\% to 12.7\% between 1997 and 2011 for the high SES category (a $5.5 \%$ increase overall) and from $13.7 \%$ to $23.8 \%$ over the same period for the low SES group (10.1\% overall).

In summary, while overweight and obesity are common across all socioeconomic groups in NSW, obesity is more common and is increasing more rapidly in lower socioeconomic groups.

The indicator covering Overweight or Obesity includes those who are overweight or obese: that is, with a Body Mass Index (BMI) of 25.0 or higher: overweight (BMI from 25.0 to 29.9) and obese (BMI of 30.0 and over).

Further information on these definitions and methods is available from the Health Statistics NSW website at: www.healthstats.nsw.gov.au/indicator/beh_bmi_age. 